\title{
Evaluación del cambio en la concentración de dióxido de carbono en una corriente de gas mediante el uso de Chlorella vulgaris
}

\author{
Iván Rodrigo Astros ${ }^{\star}$, María Camila Navarro** Adriana Inés Páez ${ }^{\star \star \star}$ \\ Fundación Universidad de América, Bogotá. Grupo de Investigación: Fotocatálisis. \\ Línea de investigación: Biorremediación
}

FECHA DE ENTREGA: 30 DE NOVIEMBRE DE 2013

FECHA DE EVALUACIÓN: 13 DE MAYO DE 2014

FECHA DE APROBACIÓN: 21 DE MAYO DE 2014

\begin{abstract}
Resumen Chlorella vulgaris tiene una eficiencia de $38 \%$ en la remoción de dióxido de carbono presente una corriente de gas, el estudio se fundamento en la experimentación con especies de microalgas nativas de Colombia para su uso potencial como tratamiento en procesos industriales de combustión. El trabajo se inició con una revisión bibliográfica para identificar las condiciones de cultivo adecuadas de la microalga Chlorella vulgaris, posterior a esto se realizó el diseño básico de un biorreactor para llevar a cabo el cultivo y determinar la cinética de crecimiento de la microalga, después se propuso el diseño de experimentos que incluye dos factores: Concentración de dióxido de carbono $\left[\% \mathrm{CO}_{2} \mathrm{v} / \mathrm{v}\right]$ y duración del fotoperíodo; se realizaron cuatro tratamientos para verificar el comportamiento de las variables por acción de la microalga en diferentes condiciones. Finalmente se analizaron los resultados y se determinó el tratamiento más eficiente. Los resultados muestran que el tratamiento más eficiente es aquel en el que los factores tienen los mayores valores, es decir, el fotoperíodo mas largo y la mayor concentración de $\mathrm{CO}_{2}$.
\end{abstract}

\begin{abstract}
Chlorella vulgaris is $38 \%$ efficient in removing the carbon dioxide that is present in a gas stream. This study was based on experiments carried out with species of microalgae native from Colombia to be used in industrial processes of combustion. We started with a bibliographical review in order to identify the adequate culture conditions of the microalgae Chlorella vulgaris. After this, we designed a bioreactor to do the culture and determine the growth kinetics of the microalgae. Also, we proposed the design of experiments that included two factors: concentration of carbon dioxide $\left[\% \mathrm{CO}_{2} \mathrm{v} / \mathrm{v}\right]$ and photoperiod length. We carried out four treatments to verify the variable behavior by means of the microalgae in different conditions. Finally, we analyzed the results and determined the most efficient treatment, which, according to the results, is the one where
\end{abstract}

\footnotetext{
* Estudiante de pregrado de Ingeniería Química. ivan.astros@gmail.com

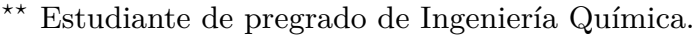

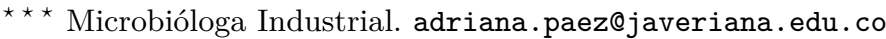


the factors have greater values. This means, the longest photoperiod and the largest concentration of $\mathrm{CO}_{2}$.

Palabras Clave: biorreactor, cinética de crecimiento, combustión, Chlorella vulgaris, dióxido de carbono, fotoperiodo.

Keywords: bioreactor, growth kinetics, combustion, Chlorella vulgaris, carbon dioxide, photoperiod.

\section{Introducción}

Las emisiones de $\mathrm{CO}_{2}$ generadas por diversas industrias, y los efectos provocados sobre el calentamiento del planeta, promueven el desarrollo de tecnologías innovadoras para controlar las emisiones de este gas de efecto invernadero. El empleo de microorganismos como biorremediadores en las industrias que emiten gases de combustión a la atmósfera tiene como fin proponer una solución, ya que la mayoría de procesos requiere de un combustible fósil, el cual genera una serie de emisiones tóxicas y contaminantes, principalmente dióxido de carbono como resultado de la oxidación de los combustibles durante la combustión.

\section{Metodología}

\section{Microalgas}

Las microalgas fuerón obtenidas de la Universidad Nacional de Colombia la cual presenta la clasificación del cuadro 1 .

\begin{tabular}{ll}
\hline Reino & Plantae \\
Subreino & Thalophyta \\
Phylum & Chlorophyta \\
Clase & Chlorophyceae \\
Orden & Chlorococales \\
Familia & Chloropitae \\
Género & Chlorella vulgaris \\
\hline
\end{tabular}

Cuadro 1. Clasificación científica para Chlorella vulgaris. Fuente: [1]. Modificado por los Autores.

\subsection{Cultivo de Microalgas}

Para el cultivo se establecio según [1], factores que inciden en el crecimiento del microorganismo; algunos de los factores importantes para llevar a cabo el cultivo de microalgas se presentan en el cuadro 2 . 


\begin{tabular}{ll}
\hline Factor & Valor \\
\hline Luz [14] & $650-22,200$ Lux \\
Temperatura [4] $18-25^{\circ} \mathrm{C}$ \\
pH [1] & $7-8$ \\
\hline
\end{tabular}

Cuadro 2. Condiciones de cultivo.

\subsection{Condiciones de cultivo}

La microalga se cultivo con intensidad lumínica de 1200 lux medida con el luxómetro, se obtiene el $\mathrm{CO}_{2}$ de un tanque de almacenamiento desplazado por un compresor que suministra el gas al biorreactor. Se utiliza el medio de cultivo BBM, cuya composición se muestra en el cuadro 3. El pH final del medio BBM es de 6.6, no se consideró adecuado incrementar el valor del $\mathrm{pH}$ ya que la adición de un nuevo componente alteraría el medio de cultivo.

\begin{tabular}{cc}
\hline Componente & Cantidad \\
\hline \multicolumn{2}{c}{ Solución de Macronutriente } \\
\hline $\mathrm{NO}_{3} \mathrm{Na}$ & $25 \mathrm{~g} / \mathrm{L}$ \\
$\mathrm{CaCl}_{2} \cdot 2 \mathrm{H}_{2} \mathrm{O}$ & $20 \mathrm{~g} / \mathrm{L}$ \\
$\mathrm{MgSO}_{4} \cdot 7 \mathrm{H}_{2} \mathrm{O}$ & $7.5 \mathrm{~g} / \mathrm{L}$ \\
$\mathrm{HK}_{2} \mathrm{PO}_{4}$ & $7.5 \mathrm{~g} / \mathrm{L}$ \\
$\mathrm{H}_{2} \mathrm{KPO}_{4}$ & $17.5 \mathrm{~g} / \mathrm{L}$ \\
$\mathrm{NaCl}$ & $2.5 \mathrm{~g} / \mathrm{L}$ \\
\hline $\mathrm{Solución} \mathrm{de} \mathrm{Micronutriente}$ \\
\hline $\mathrm{EDTA} \mathrm{Na}_{2}$ & $50 \mathrm{~g} / \mathrm{L}$ \\
$\mathrm{KOH}$ & $31 \mathrm{~g} / \mathrm{L}$ \\
$\mathrm{FeSO}_{4} \cdot 7 \mathrm{H}_{2} \mathrm{O}$ & $4.98 \mathrm{~g} / \mathrm{L}$ \\
$\mathrm{H}_{2} \mathrm{SO}_{4}$ & $1 \mathrm{~g} / \mathrm{L}$ \\
$\mathrm{H}_{3} \mathrm{BO}_{3}$ & $11.42 \mathrm{~g} / \mathrm{L}$ \\
$\mathrm{ZnSO}_{4} \cdot 7 \mathrm{H}_{2} \mathrm{O}$ & $8.8 \mathrm{~g} / \mathrm{L}$ \\
$\mathrm{MnCl}_{2} \cdot 4 \mathrm{H}_{2} \mathrm{O}$ & $1.44 \mathrm{~g} / \mathrm{L}$ \\
$\mathrm{MoO}_{3}$ & $0.71 \mathrm{~g} / \mathrm{L}$ \\
$\mathrm{CuSO}_{4} \cdot 5 \mathrm{H}_{2} \mathrm{O}$ & $1.57 \mathrm{~g} / \mathrm{L}$ \\
$\mathrm{Co}\left(\mathrm{NO}_{3}\right)_{2} \cdot 6 \mathrm{H}_{2} \mathrm{O}$ & $0.49 \mathrm{~g} / \mathrm{L}$ \\
\hline
\end{tabular}

Cuadro 3. Composición del medio de cultivo BBM. Fuente: [18]. Modificado por los Autores. 
Se preparó un pre inóculo al cual se le realizaron pruebas de pureza para verificar la ausencia de microorganismos contaminantes. El cultivo se realizó en un biorreactor del tipo columna de burbujeo ya que es la configuración recomendada para el cultivo de algas. [6]

\subsection{Diseño del biorreactor}

Se siguió el modelo de biorreactor tipo columna de burbujeo recomendado para el cultivo de Chlorella vulgaris, en el cual la relación altura diámetro está entre 3 y 6 [6]. Debido a que se usaron $2.5 \mathrm{~L}$ de medio de cultivo y $50 \mathrm{~mL}$ de preinóculo, el volumen total del biorreactor debe ser de mínimo $3 \mathrm{~L}$, por lo cual se selecciono un diámetro interno de $10 \mathrm{~cm}$ y una altura de $32 \mathrm{~cm}$. El tiempo de retención total fue de cinco días (120 horas), ya que a este tiempo se alcanza la fase estacionaria de crecimiento celular.

El montaje que se siguió se aprecia en la figura 1. La concentración de $\mathrm{CO}_{2}$ se monitoreó con ayuda del sensor K-33 ICB.

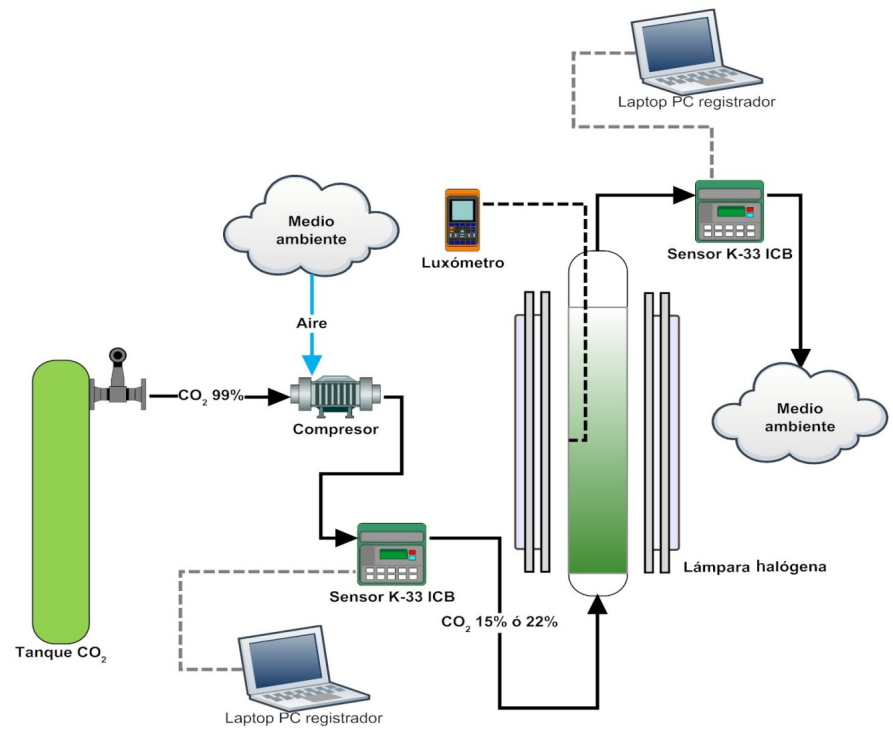

Figura 1. Esquema del montaje para el desarrollo de los tratamientos.

\section{Resultados}

El objetivo de la investigación permitió evaluar el cambio en la concentración de dióxido de carbono a causa del metabolismo del $\mathrm{CO}_{2}$ por parte de la microalga 
Chlorella vulgaris al hacer circular un gas a través del medio de cultivo. Se probaron varios conjuntos de tratamientos mediante un diseño de experimentos de dos niveles $\left(2^{K}\right)$. Los factores que se usaron fueron la Concentración de $\mathrm{CO}_{2}$, con un nivel alto $\left(22 \% \mathrm{CO}_{2} \mathrm{v} / \mathrm{v}\right)$ y bajo $\left(15 \% \mathrm{CO}_{2} \mathrm{v} / \mathrm{v}\right)$, y la duración de los fotoperíodos, con un nivel largo (16 horas de luz y 8 horas de oscuridad.) y corto (10 horas de luz y 14 horas de oscuridad).

La cinética de crecimiento del alga siguió un modelo logístico en el cual se observa que a medida que el tiempo pasa la curva adquiere una tendencia lineal a un tamaño de población determinado (1,1E7 Células $/ \mathrm{mL}$ ). Este tipo de comportamiento durante los tratamientos indica que no se sobrepasaron las condiciones de cultivo máximas que puede soportar Chlorella vulgaris [14].

De la ecuación 1 del modelo logístico se puede obtener el valor máximo de la velocidad de crecimiento relativa al tiempo $(\mu)$ de $0.0029 h^{-1}$.

$$
C . V .=\frac{1.1 \times 10^{7} \cdot 2.15 \times 10^{6} \cdot e^{0.0029 t}}{1.1 \times 10^{7}+2.15 \times 10^{6} \cdot e^{0.0029 t}-1}
$$

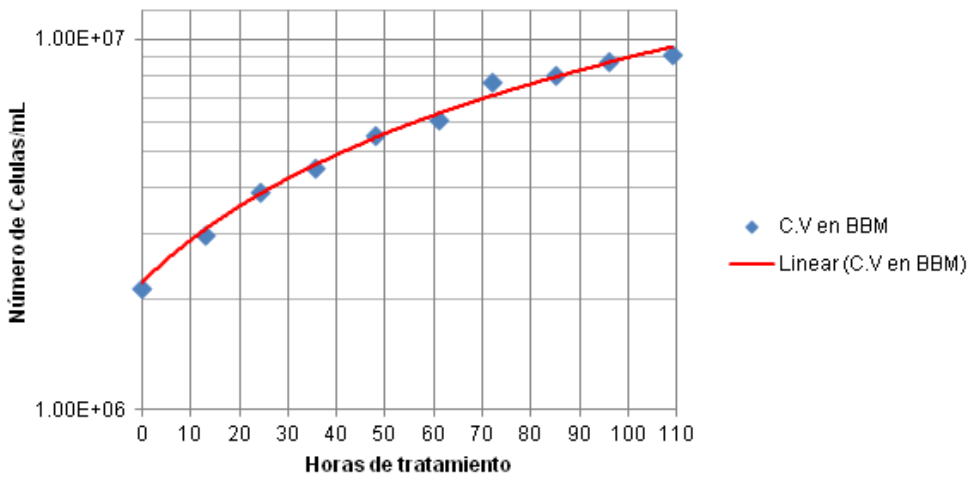

Figura 2. Curva general de crecimiento para Chlorella vulgaris en medio BBM

El mejor tratamiento que se logró fue aquel en el cual se tenían los mayores valores de fotoperíodo y concentración de dióxido de carbono, lo cual altera el metabolismo del alga, incrementando rápidamente su población celular disminuyendo así en una mayor proporción el dióxido de carbono presente en el ambiente. En general se puede ver que el crecimiento celular es similar en todos los tratamientos, variando solo la velocidad de crecimiento durante los primeros días y alcanzando la fase estacionaria aproximadamente al quinto día.

El análisis de los factores se realizó calculando el diferencial de la concentración de dióxido de carbono a la salida del biorreactor para los tratamientos y las réplicas entre las muestras 1 y 10 . Los datos promedio obtenidos para cada 
tratamiento se encuentran reportados en figura 3. El factor fotoperíodo es el que va a afectar en mayor medida el diferencial de la concentración de dióxido de carbono a la salida del biorreactor.

\begin{tabular}{|c|c|c|c|}
\hline & \multicolumn{2}{|c|}{$\% \mathrm{CO}_{2} \mathrm{v} / \mathrm{v}$} \\
\hline & $\Delta \% \mathbf{C O}_{2}$ & $22 \%$ & $15 \%$ \\
\hline \multirow{4}{*}{ 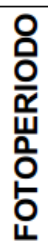 } & \multirow{2}{*}{16 Horas } & 7,655 & 7,676 \\
\hline & & 8,212 & 8,799 \\
\hline & \multirow{2}{*}{10 Horas } & 5,88 & 7,103 \\
\hline & & 6,278 & 7,936 \\
\hline
\end{tabular}

Figura 3. Resultado de los tratamientos.

Todos los tratamientos mostraron una tendencia similar en la disminución de dióxido de carbono, esta tendencia se observa en la figura 4 y se puede expresar mediante una función polinómica que se ajuste a la curva contenida en la ecuación 2. Esta similitud en la tendencia de las gráficas coincide con los resultados del análisis de varianza, en el cual la combinación de los factores no tiene una gran incidencia en la capacidad de fijación de la microalga, ya que esta capacidad de fijación está ligada directamente a la concentración celular, y al tenerse proporcionalidad en la tasa de crecimiento celular durante los diferentes tratamientos y sin importar las condiciones a las que se sometiera la microalga, la capacidad de fijación de dióxido de carbono fue de $1.55\left[\% \mathrm{CO}_{2} \mathrm{v} / \mathrm{v}\right] /$ día.

$$
\begin{aligned}
y & =-7 \times 10^{-40} x^{6}+3 \times 10^{-32} x^{5}-4 \times 10^{-25} x^{4} \\
& +4 \times 10^{-18} x^{3}-2 \times 10^{-11} x^{2}+3 \times 10^{-5} x-7.0354 \quad R^{2}=0.9987
\end{aligned}
$$

En los cuatro tratamientos se puede ver un comportamiento de "escalera" entre las diferentes muestras que se tomaron, este comportamiento se debe al metabolismo celular de Chlorella vulgaris, que consiste en un Ciclo de Calvin similar al de las plantas, con una fase luminosa y una oscura. Durante la fase luminosa, se tiene un valor constante en la tasa de fijación de dióxido de carbono y un ligero aumento en la concentración celular, ya que durante este período el alga obtiene la energía lumínica (ATP) y hace fijación del dióxido de carbono presente en el aire sin aumentar su población celular debido a que no se ha reducido el material inorgánico a material orgánico, es decir la transformación del dióxido de carbono a glucosa, por lo cual no se puede apreciar un gran cambio en el $\% \mathrm{CO}_{2}$ a la salida del biorreactor. Durante la fase oscura, el alga no hace fijación del dióxido de carbono en el aire, solo realiza división celular usando al dióxido de carbono como fuente de carbón para sintetizar azúcares, y por esta 


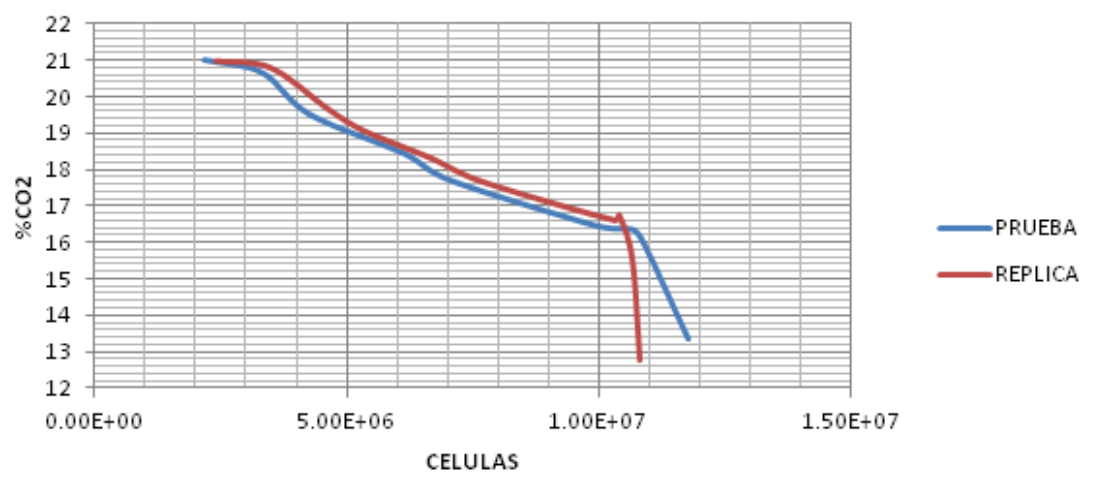

Figura 4. Resultados del primer tratamiento.

razón, al realizar la medida de la concentración de dióxido de carbono a la salida del biorreactor al inicio del nuevo fotoperíodo, se presenta un cambio abrupto en la concentración celular y en él $\% \mathrm{CO}_{2}$.

\section{Conclusiones}

- La evaluación de los tratamientos muestra que no existen diferencias significativas en la disminución de la concentración del dióxido de carbono, cuyo valor promedio fue de $6,5 \% \mathrm{CO}_{2} \mathrm{v} / \mathrm{v}$, correspondiente a un $38 \%$ de eficiencia.

- El dimensionamiento del biorreactor fue el adecuado para el desarrollo de los tratamientos, cumpliendo con los requerimientos de agitación, cantidad de luz y distribución de nutrientes necesarios para alcanzar la fase de crecimiento estacionario en un periodo de tiempo corto y una considerable disminución de dióxido de carbono.

- Los cultivos bajo las condiciones de temperatura, concentración de dióxido de carbono, medio de cultivo e intensidad lumínica, alcanzaron la concentración celular necesaria para la disminución del dióxido de carbono comparable a la obtenida por otros investigadores.

- La cinética de crecimiento sigue un modelo logístico bajo condiciones de operación diferentes, teniéndose una reproducibilidad del comportamiento aunque algunas diferencias en la concentración máxima de acuerdo al fotoperiodo de luz usado.

- La capacidad de fijación de dióxido de carbono no presento diferencias significativas bajo las diferentes condiciones de operación, confirmando que los factores intensidad de luz y concentración de dióxido de carbono no influyen de forma directa en la capacidad de fijación y que esta capacidad está determinada en mayor proporción por la concentración celular. 
- Estudios relacionados, reportan para tratamientos similares una disminución del $10 \%$,que es mayor a lo obtenido, debido a que el medio de cultivo, fue suplementado con nutrientes, que mejoraron el crecimiento microalgal y por ende la velocidad en la fijación del $\mathrm{CO}_{2}$.

\section{Agradecimientos}

A Adriana Páez, Microbióloga Industrial, y Directora de este proyecto, ya que gracias a su guía y apoyo se logro desarrollar con éxito este trabajo.

A la Universidad de América por darnos los conocimientos necesarios para obtener el título de Ingenieros Químicos y desarrollar este proyecto.

Por último agradecemos a nuestras familias y todos aquellos que nos han acompañado a lo largo del desarrollo de este trabajo.

\section{Referencias}

1. Abalde J., Cid A., Fidalgo J. P., Torres E., Herrera C.: Microalgas: Cultivo y aplicaciones. Coruña: Universidad de la Coruña, 210p. (1995)

2. Agencia de protección ambiental de Estados Unidos.: Guía del Medio Ambiente de la EPA. Documento No. 520/B-94-001. Washington, DC: La agencia. (1999)

3. Bei, W.: CO2 bio-mitigation using microalgae. En: Apply Microbiological Biotechnology. Mayo, vol.79, p. 707-718 (2008)

4. Chaparro De Barrera, A.: Curso de fisiología vegetal. Bogotá: Universidad Nacional de Colombia. 52p. (2010)

5. Chaumont, D.: Biotechnology of algal biomass production: A review of systems for outdoor mass culture. En: Journal of apply Phycology. Agosto, vol.1, p. 593-604 (1993)

6. Chisti Y., Murray Moo-Young.: Bioreactors, Encyclopedia of Physical Science and Technology. En: Academic Press. vol. 2, p. 247-266 (2002)

7. Gobierno De Navarra.: Calidad del aire, Dióxido de carbono [En línea]. Disponible en: http://www.navarra.es/home_es/Temas/Medio+Ambiente/Calidad+del+ aire/Informacion/Contaminantes/C02.htm [citado Abril 06, 2011]

8. Grobbelaar, J.: Physiological and technological considerations for optimizing mass algal cultures. En: Journal of apply Phycology. Octubre, vol. 12, p. 201-206 (2000)

9. Guevara L, E.: Diseño, construcción y caracterización hidrodinámica de un biorreactor multifuncional. Trabajo de grado Ingeniería de Alimentos. Huajuapan de Lean: Universidad tecnológica de la mixteca. Facultad de Ingeniería, 107p. (2004)

10. Instituto Colombiano de Normas Técnicas Y Certificación.: Documentación. Presentación de tesis, trabajos de grado y otros trabajos de investigación. NTC 1486. Sexta actualización. Bogotá: ICONTEC, 2008, 110 p. (2008)

11. Javanmardian M., Palsson B.: High-density photoautotrophic algal cultures: Design, construction, and operation of a novel photobioreactor system. En: Biotechnoly Bioengineering. Diciembre, 1991, vol. 38, p. 1182-1189 (1991)

12. Li Y., Horsman M., Wu N., Lan CQ., Dubois-Calero N.: Biofuels from microalgae. En: Biotech Prog. ASAP Article. Julio, 2008, vol. 4, p. 815-820 (2008)

13. Matsumoto $\mathrm{H}$.: Influence of $\mathrm{CO} 2, \mathrm{SO} 2$ and $\mathrm{NO}$ in flue gas on microalgae productivity. En: Journal Chemical Engineering Japan, 1997, vol. 30, p. 620-624 (1997) 
14. Pearsall W. H., Loose L.: The Growth of Chlorella Vulgaris in pure culture. En: Royal Society of London. Series B, Biological Sciences, 1936, Vol. 121, p. 451-501 (1936)

15. Pirt, John.: A tubular bioreactor for photosynthetic production of biomass from carbon dioxide: design and performance. En: Journal of Chemical Technoly \& Biotechnoly. Enero, 1983, vol. 33, p. 35-58 (1983)

16. Pirt, John.: The photosynthetic efficiency of Chlorella biomass growth with reference to solar energy utilization. En: Journal of Chemical Technoly \& Biotechnoly. Agosto, 1980, vol. 30, p. 25-34 (1980)

17. Skjanes K., Lindblad P., Muller J.: Bio CO2-a multidisciplinary, biological approach using solar energy to capture $\mathrm{CO} 2$ while producing $\mathrm{H} 2$ and high value products. En: Biomolecular Engineering. Mayo, 2007, vol. 24, p. 405-413 (2007)

18. Stein, J. R.: Handbook of Phycological Methods, Culture Methods and Growth Measurements. Cambridge: Cambridge University Press, 1973. 460p. (1973)

19. Usui N., Ikenouchi M.: The biological CO2 fixation and utilization project by RITE (1): highly-effective photobioreactor system. En: Energy Conversion Management. 1997, vol. 38, p. 487-492. (1997)

20. Verlaan P., Vos J-C., Van't Riet K.: From bubble column to air-lift-Loop Reactor: Hydrodynamics and Axial Dispersion of the Transition Flow Regime, 2nd International Conference on Bioreactor Fluid Dynamics. En: Elsevier Applied Science Publishers. Enero, 1988, p. 259-261 (1988)

21. Yeoung, S. Y., Jong, M. P.: Development of gas recycling photobioreactor system for microalgal carbon dioxide fixation. En: Korean Journal of Chemical Engineering. Septiembre, 1997, vol. 4, p. 297-300 (1997) 